\title{
A lab-on-a-chip device for investigating the fusion process of olfactory ensheathing cell spheroids
}

\author{
Ahmed Munaz $^{\mathrm{a}}$, Raja K. Vadivelu ${ }^{\mathrm{b}}$, James A. St John ${ }^{\mathrm{b}}$ and Nam-Trung Nguyen ${ }^{\mathrm{a} *}$
}

Understanding the process of fusion of olfactory ensheathing cell spheroids will lead to improvement of cell transplantation therapies to repair spinal cord injuries. The successful fusion of transplanted spheroids will enable alternative transplantation strategies to be developed for in-vivo applications. This paper describes the use of a microfluidic device to trap and fuse olfactory ensheathing cell spheroids. The velocity, the pressure distribution in the device were simulated numerically to predict the trapping location. The simulation predicted the optimum flow rates for trapping the spheroids in the later experiments. Simulated particle trajectories were verified experimentally with tracing of fluorescent micro particles. The fusion process of the spheroids was investigated over a period of 48 hours. The microfluidic platform presented here can be used for testing potential drugs that can promote the fusion process and improve the transplantation therapy.

\section{Introduction}

Olfactory ensheathing cells (OECs) are neuroglia cells that provide support and protection for olfactory sensory neurons in the peripheral nervous system (PNS). ${ }^{1}$ OECs direct olfactory axons of the sensory neurons from the olfactory mucosa that lines the nasal cavity to their target site in the olfactory bulb within the central nervous system (CNS). ${ }^{2}$ The olfactory system continually regenerates with new olfactory sensory neurons arising from stem cells that line the olfactory epithelium. As the new neurons extend axons up to the olfactory bulb, OECs guide and support their growth. OECs release diffusible factors such as basic fibroblast growth factor (bFGF), nerve growth factor (NGF), brain derived neurotropic factor (BDNF), insulin like growth factor 1 (IGF-1), extra cellular matrix proteins such as laminin, and fibronectin that promote axon growth. ${ }^{3-5}$

Considering their unique characteristics, the transplantation of OECs has a potential therapeutic application for the repair of neural injuries in CNS or PNS caused by accidents, strokes or Parkinson's disease. For instance, Tabakow et al. assessed the feasibility of the OEC transplantation for patients with complete spinal cord injury. ${ }^{6}$ Microinjections of dissociated cell suspensions were performed into the damaged site. Functional motor and sensory improvements were observed in one recipient a year after the transplantation. This proof-of-principle result demonstrates that the therapy can work in humans. However transplanting OECs to the target region and survival of cells post-transplantation are still a potential challenge. The hostile environment of the transplantation region results in a large loss of the transplanted cells, especially when dissociated cell suspensions are used.

Recently, three-dimensional spheroid culture emerged as a promising method that can be used to improve cell characteristics relevant for neural repair therapies. ${ }^{7}$ For example, Chi et 
al. showed an efficient differentiation of schwann cells (SCs) from multipotent adipose stem cell spheroids in an in vitro model. ${ }^{8}$ The induced SCs were transplanted into a rat spinal cord injured (SCI) site that successfully engrafted and formed myelin sheath on the CNS axons. The transplanted spheroid validated a rapid and efficient repair of damaged CNS/PNS. Tseng et al. authenticated the surface derived mesenchymal stem cell spheroids (MSCs) with higher differentiation capabilities, engrafting potential and functional recovery then dispersed single cells. ${ }^{9}$ MSCs were mixed with the nerve conduit to repair a $10 \mathrm{~mm}$ gap of rat sciatic nerve. The study showed a short recovery time of less then 21days. Larger nerve regeneration and improved gene expression offer an improved source for cell based therapy. Jeong et al. introduced a hemispherical microchannel array system to generate 3D nerve neural bundles from neuro spheroids. ${ }^{10}$ Neural progenitor cells spontaneously aggregated to form the spheroids in the deep micro well design. They networked to neighbouring spheroids through newly generated neurite strands and differentiated into glial and neuronal cells.

A uniform spheroid mimics the living environment more closely compared with cells cultured as a monolayer and therefore 3D spheroids offer advantages for in-vitro drug screening and neural repair therapies. ${ }^{11}$ Moreover, self-assembled spheroids promote uniform geometry and structural properties, which are optimal for cell-matrix interactions and improved transplantation therapy for neural repair. Thus, to improve transplantation approaches and drug development, the functional unit of a spheroid shows more clinical importance than single cell approaches. ${ }^{12,13}$ We previously used floating liquid marble as an efficient micro bioreactor for the formation of OECs spheroids. ${ }^{7}$ The liquid marble provides a confined space in which cells are free to interact effectively with each other. ${ }^{12}$ The formation of the spheroid depends on the interaction of cells with each other. Furthermore, effective and in-vivo like cell-cell communication leads to the formation of multiple spheroids. ${ }^{14}$

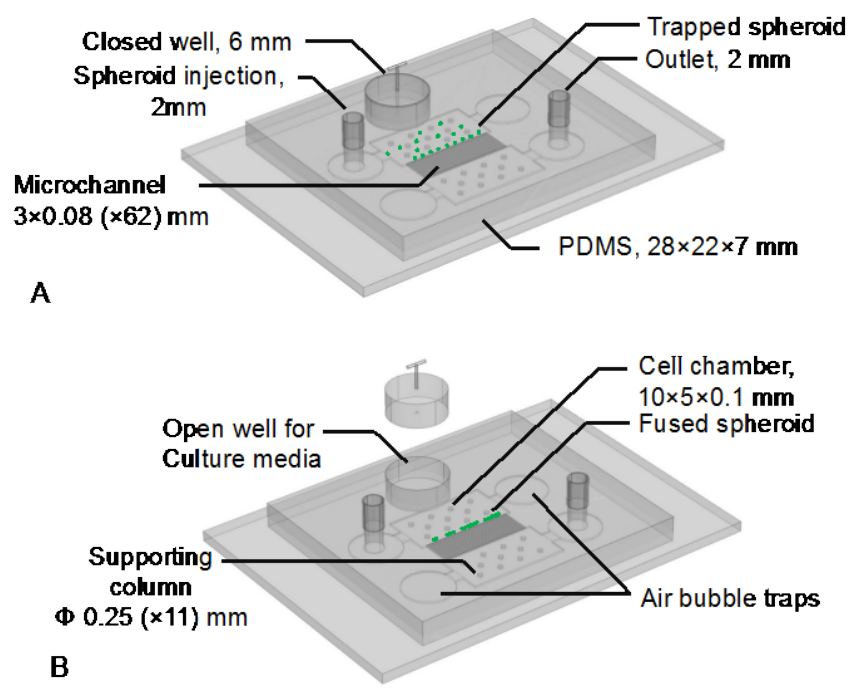

Fig. 1 Schematic of the lab-on-a-chip device in the two modes: (A) trapping mode; and (B) fusion mode.

In the present work, we designed and fabricated a microfluidic lab-on-a-chip (LOC) device as a test bed for the survival of the OEC spheroids previously cultured in the liquid-marble 
bioreactors. A liquid marble (LM) is a drop of liquid, coated with hydrophobic powder such as polytetrafluoroethylene (PTFE). ${ }^{15}$ The deformable and non-adhesive coating with the fine pores from the floated marble provides necessary gas exchange that is amenable for culturing multiple spheroids. Moreover, the microfluidic system improves the investigation by lowering the required volume of fluid sample, and offering a observable miniature 3D environment. ${ }^{16}$ The devices were made of poly(dimethylsiloxane) (PDMS) which is easy to fabricate and compatible with most biological assays. The PDMS device offers elastomeric property, low toxicity and high gas permeability which are suitable for cells and spheroids to grow. ${ }^{17}$ Such LOC devices are able to provide appropriate low cost micro-environments for screening cell spheroids, tissues and small model organisms. ${ }^{18}$ The diameters the OEC spheroids grown with our technology are between $80-120 \mu \mathrm{m}$, which is well suited to be handled in these devices.

Our objective is to introduce a LOC device that facilitates the fusion of the cell spheroids. We investigated the fusion process of the OEC spheroids in the microfluidic environment, as this process represents the formation of complex tissue structures. Cell fusion is a self-assembly process where multiple spheroids contact and coalesce to form a single structure. Cell migration, cell-cell interactions, and cell-matrix interactions are the major factors mediating spheroid fusions. ${ }^{19-21}$ Elucidating how to manipulate these processes will advance the potential therapeutic use of spheroids. Most of the previously reported microfluidic devices have been utilised to culture spheroids with different cell types. For instance, Fukuda et al. developed a micro fabricated chip that could form hepatocyte spheroids in a row of microchannels. $^{22}$ Primary hepatocytes were seeded onto the micro-well to form spontaneous uniform spheroids. Hiroki et al. introduced a micro rotational fluid flow inside of a microfluidic device to construct controllable sized 3D hepatocyte spheroids. ${ }^{23}$ The fluid flow also provided ample nutrient and oxygen for the spheroid survival. Anada et al. developed a PDMS multi-chamber device for the formation of multiple spheroids. ${ }^{24}$ The setup decompresses the PDMS membrane that allows the deposited MG63 and HepG2 cells to form spheroids within a day.

To our best knowledge, culturing OEC spheroids in a liquid marble, transferring them into a microfluidic device using hydrodynamic force and fusing them in-vitro have not been conducted and analysed before. The present paper reports how the multiple OECs spheroids are trapped and fused over a time period inside of a micro-fluidic chamber. The paper analyses and discusses possible modification for the injection and further improvement of the device design. 

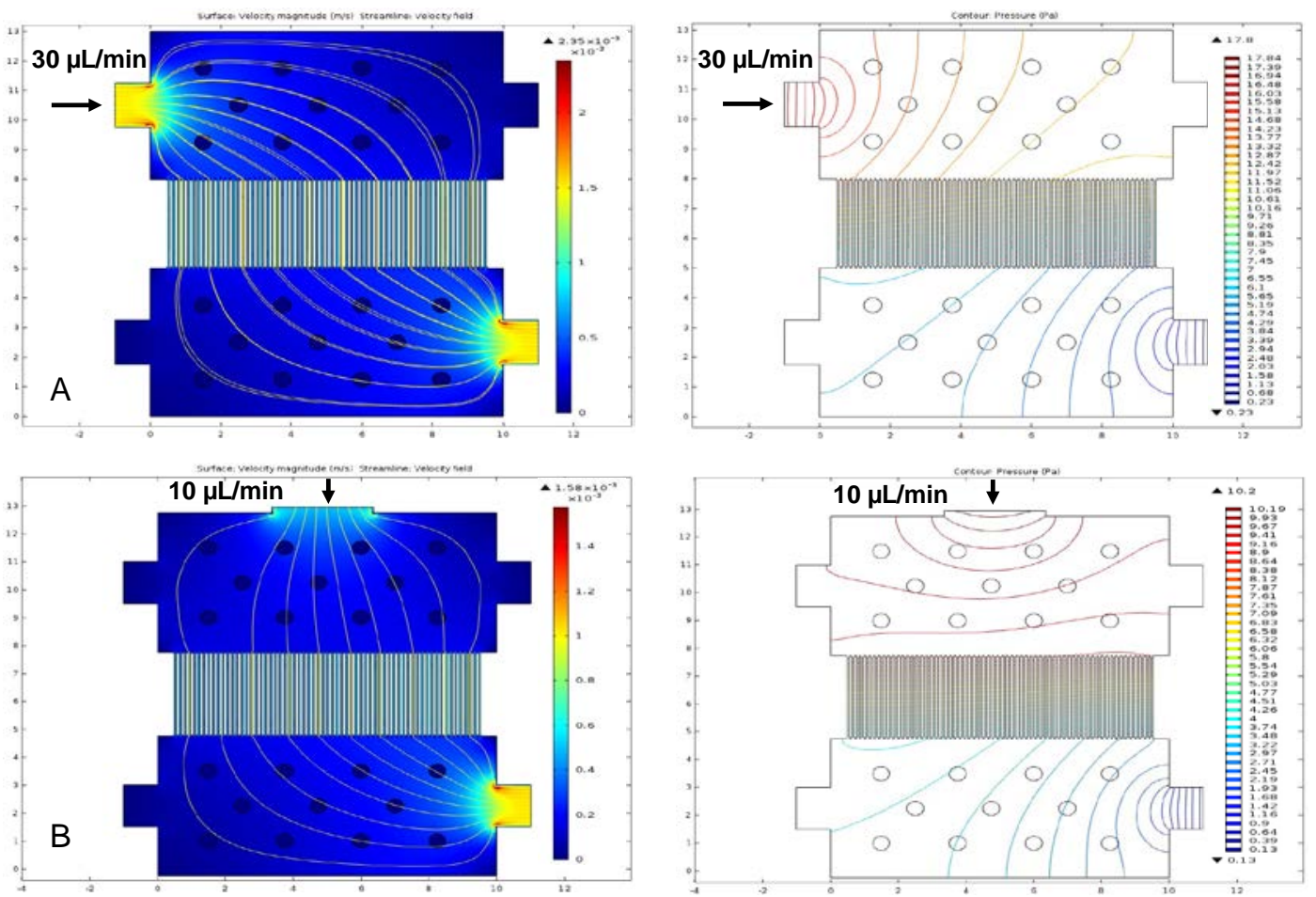

Fig. 2 Numerical analysis of the flow field and pressure field inside of the micro fluidic devices: (A) Trapping mode with the well for culture media closed; and (B) Fusion mode with the inlet closed (Units in $\mathrm{ms}^{-1}$ for velocity, Pa for pressure and $\mathrm{mm}$ for spatial units)

\section{Fabrication and optimisation of the lab-on-a-chip device}

\section{Materials and methods}

We developed a micro fluidic device containing an array of microchannels as the trapping bed for the OEC spheroids. The microchannels array connects two large chambers, one of which serves as the culture chamber for the fusion process. The basic device contains an inlet attached with one chamber and an outlet to another one. The OEC spheroids are expected to be trapped above the microchannels array. Eleven supporting pillars were placed in the chambers to prevent them from collapsing. The device was designed using CleWin 3.0 software and plotted on a $28-\mathrm{mm} \times 22-\mathrm{mm}$ plastic mask (IGI-sing, Singapore). The fabrication of the microfluidic device involved a two-step soft lithography process. The master mould was made of SU8. The trapping array consists of 62 microchannels with a length of $3000 \mu \mathrm{m}$ and a width of $80 \mu \mathrm{m}$. The microchannels are placed $60 \mu \mathrm{m}$ apart from each other. The actual PDMS devices were replicated using the SU8 master mould. The detailed fabrication process is described as follows. 

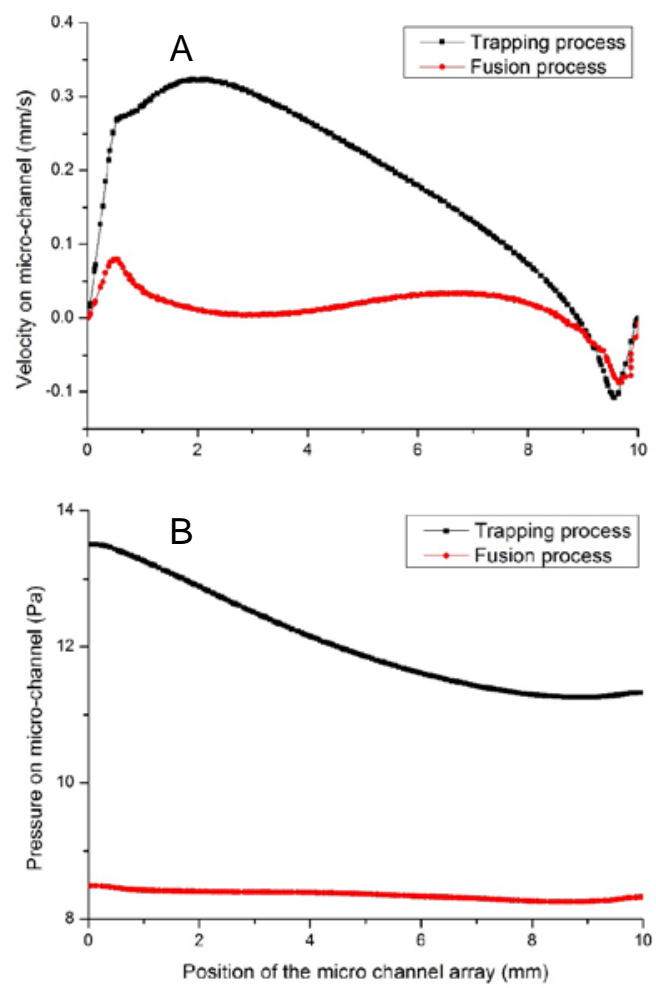

Fig. 3 Numerically simulated velocity and pressure distribution on top of the microchannel array for both trapping and fusion modes: (A) Velocity; (B) Pressure.

A layer of photoresist SU-8 50 was spin coated at $3300 \mathrm{rpm}$ onto a clean silicon wafer. The SU-8 was soft baked through a series of steps $\left(65^{\circ} \mathrm{C}\right.$ for $10 \mathrm{~min}, 95^{\circ} \mathrm{C}$ for $30 \mathrm{~min}, 50{ }^{\circ} \mathrm{C}$ to cool down). Subsequent UV exposure with a dose of $920 \mathrm{~mJ} / \mathrm{cm}^{2}$ was followed by a post bake step ( $65{ }^{\circ} \mathrm{C}$ for $1 \mathrm{~min}, 95{ }^{\circ} \mathrm{C}$ for $10 \mathrm{~min}$ ). The exposed SU-8 was developed with 1methoxy-2-propanol acetate for 15 minutes. The thickness of each fluidic chamber and the channel was $100 \mu \mathrm{m}$. Polydimethylsiloxane (PDMS) pre-polymer and its cross-linker were mixed with 10:1 volume ratio and then poured on the mould, degassed and cured in an oven at $80{ }^{\circ} \mathrm{C}$ for one hour. After peeling off the cured PDMS from the mould, 2-mm holes were punched to define the inlet and outlet ports. To inject additional media without flushing the trapped spheroids, another hole of 6-mm diameter was punched next to the culture chamber. This 6-mm opening was later used as a well for the culture media. The PDMS part was removed in a way that it could be later pushed back to close the opening during the injection, and be opened again for the supply of fresh culture media to the cells.

The well was closed during the injection of spheroids through the inlet ports. The operation mode of the device is called the trapping mode. Later the well was opened and the inlet was closed for the media flow for assisting the residual spheroid trapping. The process is called the fusion mode. The PDMS part was cleaned with isopropanol, rinsed in deionised water, treated with oxygen plasma for 45 seconds, and subsequently bonded to a glass slide. Furthermore, the firm bond was ensured with tempering in an oven at $80{ }^{\circ} \mathrm{C}$ for one hour. Fig. 
1 shows the schematic operation of the lab-on-a-chip (LOC) device. The well is closed for the trapping mode, and opened for the fusion mode.

The internal volume of the fluid in the main culture chamber was approximately $40 \mu \mathrm{L}$. A short silicon tube of 2-mm diameter was attached to the inlet and outlet ports for a leak-free fluidic delivery to and from the device.

\section{Numerical analysis}

COMSOL Multiphysics 5.2 was utilised to analyse the laminar fluid flow including velocity and pressure distribution in the trapping and culture chamber. The geometric parameters of the simulated device were the same as of the fabricated one. We selected water as the fluid in the simulation.

Initially, a flow rate of $30 \mu \mathrm{L} / \mathrm{min}$ was applied to the inlet region to investigate the velocity and pressure distribution inside the culture chamber. The media well is closed during the trapping process. Fig. 2A shows the representative velocity and pressure distribution inside the device in the trapping mode. The high velocity can be seen mainly on the inlet and outlet joints connected with the top and bottom chambers. The pressure has a relatively uniform distribution inside of the top and bottom chamber.
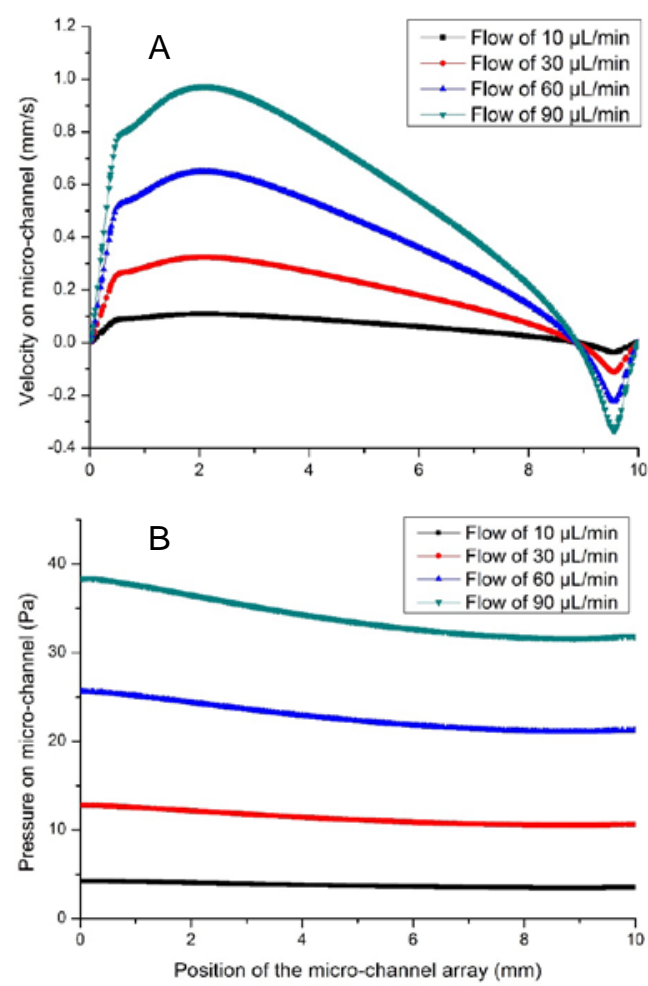

Fig. 4 Numerically simulated (A) velocity and (B) pressure distribution on top of the microchannel array for trapping mode with different inlet flow rates. 
Fig. 2B shows the media flow delivered to the trapped zones on the connecting microchannels. The top opening serves as a well for culture media. A relatively low flow rate of $10 \mu \mathrm{L} / \mathrm{min}$ was applied. As the flow field in this mode directly impacts on the trapped spheroids, a low media flow is desirable so that the spheroids are not flushed through the channel array. Moreover, as shown the later experiment, this media flow continues to trap freely floating spheroids on top of the microchannel array.

Fig. 3 shows the detailed velocity and pressure distribution on top of the microchannel array for both trapping and fusion modes. Fig. 3A indicates a gradual reduction of velocity over the trapped zone in the trapping mode. The high velocity and the high pressure next to the inlet increase the possibility of the spheroids to be flushed through the microchannel. As the velocity decreases with increasing distance from the inlet, the chance of trapping on the right side becomes higher. Once the spheroids are trapped and block the microchannel on the right side, the velocity on the left will further increase and flush the spheroids through. Thus, we expect that cell spheroids be reliably trapped on the right of the culture chamber.

Fig. 3B indicates that the trapping mode has a relatively higher pressure than the fusion mode. This over pressure allows the flow field to trap and align the spheroids over the microchannel region. The lower the inlet pressure, the higher is the chance for the spheroid to be trapped over the microchannel array. Furthermore, maintaining a low pressure for media injection reduces the chance of the spheroids being flushed through the microchannel. The lower pressure facilitated by the opening well also reduces the shear rate and provides a better environment for the interaction between cell spheroids, promoting the fusion process.

Fig. 4 shows the velocity and pressure distribution on top of the microchannel array for flow rates ranging from 10 to $90 \mu \mathrm{L} / \mathrm{min}$ in the trapping mode.

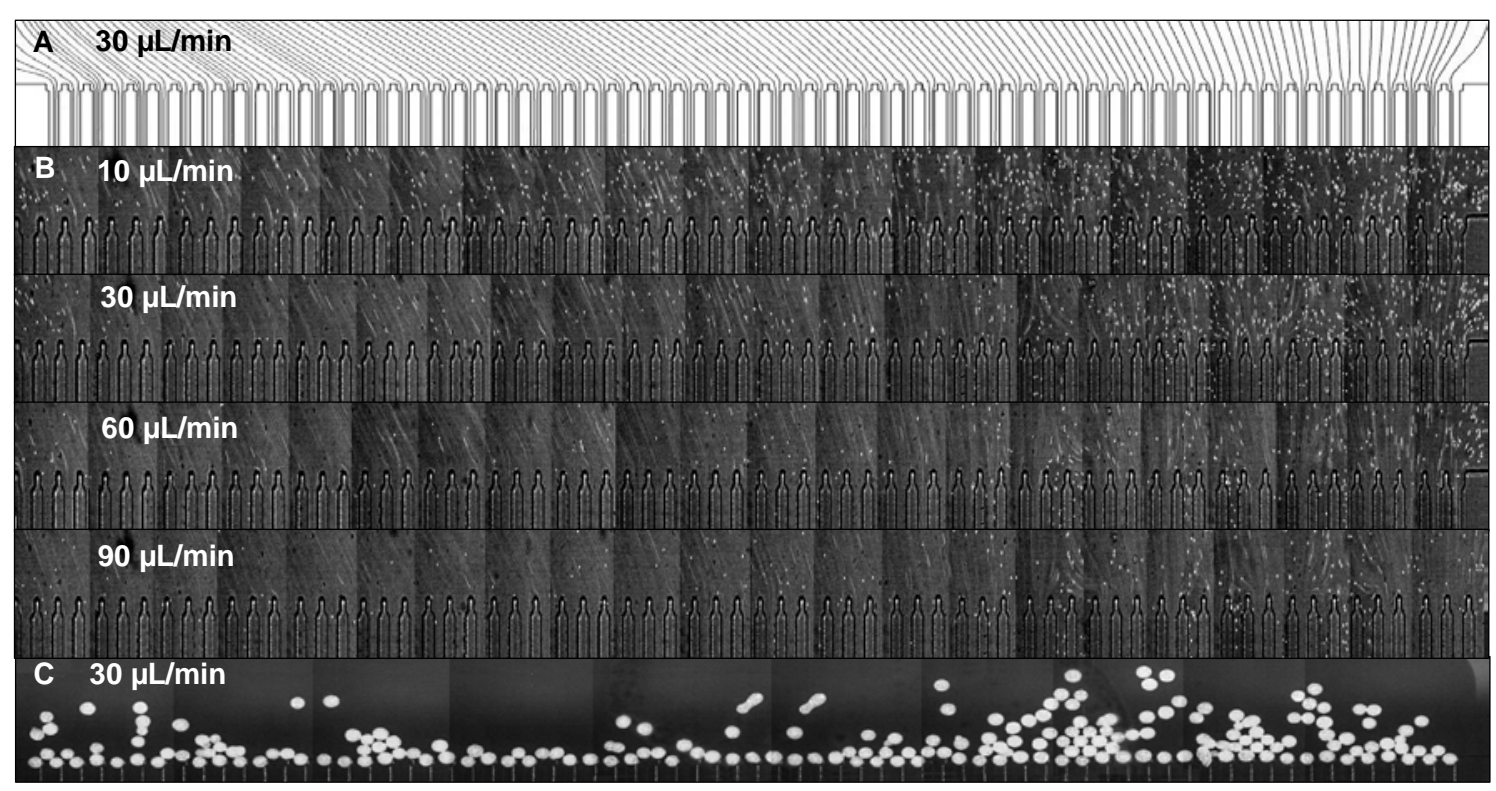

Fig. 5 Flow field and trapping demonsration: (A) numerical particle tracing with optimum flow rate of $30 \mu \mathrm{L} / \mathrm{min}$; (B) Particle tracing with 3.1- $\mu \mathrm{m}$ polymer microspheres inside of the culture chamber with different flow rate (composite photos of at least 20 frames were taken on 10X zoom in the trapping mode); and (C) Polymer microspheres with 90-105 $\mu \mathrm{m}$ of diameters at $30 \mu \mathrm{L} / \mathrm{min}$.

As the flow rate increases, the associated velocity and pressure also increase. The graphs also 
indicate a gradual reduction of velocity and pressure over the trapped zone. As the cell spheroids are deformable, the high velocity and pressure allow them to squeeze through the channel. For example, in the flow rate range of 60 to $90 \mu \mathrm{L} / \mathrm{min}$, the associated velocity and pressure are approximately $0.65-0.95 \mathrm{~mm} / \mathrm{s}$ and $26-38 \mathrm{~Pa}$, respectively. The threshold velocity and pressure for spheroid trapping are around $0.25 \mathrm{~mm} / \mathrm{s}$ and $15 \mathrm{~Pa}$ at a flow rate of $30 \mu \mathrm{L} / \mathrm{min}$, for the maximum number of trapped spheroids. The lower the inlet pressure, the higher is the chance for the spheroid to be trapped over the microchannel array. Our experiments suggested that a high pressure at high flow rates of more than $60 \mu \mathrm{L} / \mathrm{min}$ squeezes the spheroid and eventually flushes it through the microchannel array. Therefore, the flow rate of $30 \mu \mathrm{L} / \mathrm{min}$ for the trapping mode was selected.

Before the actual trapping and fusion experiments with OEC spheroids, particle tracking was performed numerically and experimentally. COMSOL particle tracking was initiated with 150 particles (diameter of 3.1- $\mu$ m each) for a flow rates ranging from 10 to $90 \mu \mathrm{L} / \mathrm{min}$ (Supplementary Fig S1). Appropriate time ranges were selected for the particle to pass through the entire device. Fig 5A show the representative simulated trajectories at the trapping zone at a flow rate of $30 \mu \mathrm{L} / \mathrm{min}$. Figure 5A indicates that the trajectory is more angled at the left side compared to the right side of the microchannel array. With increasing distance from the inlet, the streamlines become more perpendicular. This behaviour is caused by the distributions of velocity and pressure shown in Fig. 3A and 3B. As the velocity reduces with distance, the particle faces less horizontal hydrodynamic forces resulting in a more perpendicular streamline. This trend continues with the higher flow rate (Fig S1). Even though the trajectory seems identical, increasing the flow rates result in higher velocity and pressure across the microchannel array. In the actual spheroids injection process, a minimum flow rate can promote better trapping.

\section{Experimental particle tracing}

To validate the simulation results, we performed flow tracing with green fluorescent microspheres (green fluorescent, polystyrene divinlybenzene, ThermoScientific Inc.) with a diameter of $3.1 \mu \mathrm{m}$. For this purpose, deionised (DI) water and microspheres were mixed and injected with a flow rate from 10 to $90 \mu \mathrm{L} / \mathrm{min}$. A syringe (Terumo, $5 \mathrm{~mL}$ ) was mounted on an automated syringe pump (SPM 100, Simtech, Singapore) and connected with the inlet tubing of the device. The image was observed with an optical fluorescence microscope (Nikon ECLIPSE TS100) and captured with an USB camera (Edmund Optics Inc., Singapore). A video was recorded (camera software, uEye cockpit, IDS, Germany) at a rate of 30 frames per second (fps). In the subsequent step, the images of different locations are assembled into a composite image covering the entire microchannel array for the final analysis.

Fig. 5B shows the images above the microchannel array at different flow rates for the particle size of $3.1 \mu \mathrm{m}$. At a low flow rate of $10 \mu \mathrm{L} / \mathrm{min}$, the particles at the left side have a higher velocity then those at the right side as indicated by the longer particle streaks. This trajectories are angled on the left, and with increasing distance from the inlet it became perpendicular as the velocity gradually decreases. Due to the channel wall at the end, some of 
the particles reverse back. The same phenomena were observed for the flow rates of 30, 60 and $90 \mu \mathrm{L} / \mathrm{min}$, Fig. 5B. However, as flow increases, the higher particle velocity near the inlet causes longer streaks. Shorter particle streaks at the middle region and the right region indicate lower velocities. As the flow rate increases, the region with low velocities clearly shifts from left to right. Both numerical and experimental data suggested that a flow rate between $10-30 \mu \mathrm{L} / \mathrm{min}$ should serve the purpose of the spheroidal injection, where optimal trapping is ensured.

To further investigate the trapping ability, green fluorescent microspheres (Polyethylene microspheres $1.00 \mathrm{~g} / \mathrm{cc}$, Cospheric) with a diameter of 90-105 $\mu \mathrm{m}$ was injected with a flow rate of $30 \mu \mathrm{L} / \mathrm{min}$. Figure 5C shows the identical trapping pattern of trapping of cell spheroids, which is performed next. Most of the particle tended to settle at the end of the culture chamber due to the weaker hydrodynamic forces. However, as the particles are uniform and they do not pass through the channel, it was not possible to deliver the particle for longer period of time. The particle would than be trapped fill the entire culture chamber.

Formation of the olfactory spheroids

\section{Materials and method}




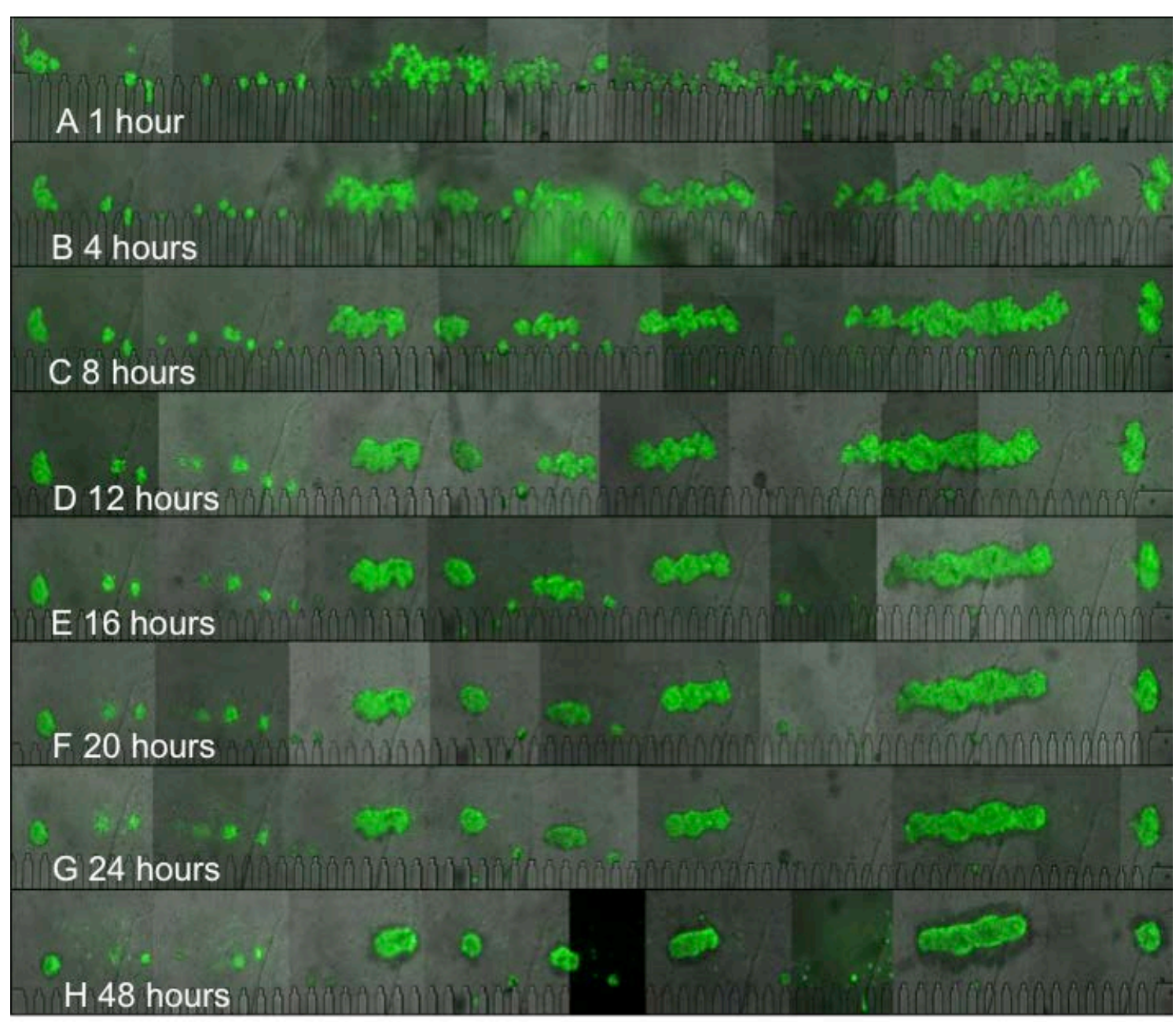

Fig. 6 Fused OECs spheroids inside of the microfluidic chamber after (A) 1 hour; (B) 4 hours; (C) 8 hours; (D) 12 hours; (E) 16 hours; (F) 20 hours; (G) 24 hours and (H) 48 hours (composite image of at least nine frames).

Desired OEC cells were harvested every 2 to 3 days after $70-80 \%$ of confluency. Uniform sized spheroids were generated with a seeding of 5000 cells per $10 \mu \mathrm{L}$ floating liquid marble. The formulation and optimization of the spheroids were reported previously. ${ }^{7}$ Cells and spheroids were cultured in OEC media consisting of DMEM/F12 (Life Technologies) supplemented with 10\% FBS, $2 \mu \mathrm{M}$ forskolin (Sigma), $20 \mu \mathrm{g} / \mathrm{mL}$ bovine pituitary extract (Gibco), 10 ng/mL FGF-2 (Pepro Tech), 10 ng/mL EGF (Pepro Tech), and 0.5\% (vol/vol) gentamicin (Life Technologies).

Polytetrafluoroethylene (PTFE) powder with particle diameter of $1 \mu \mathrm{m}$ were acquired from Sigma-Aldrich (product number 430935). The culture media droplets with the predetermined number of OECs were deposited with a micropipette onto the powder bed prepared inside a Petri dish. A gentle circular motion of the dish allowed the particle to cover the surface of the droplets. The formed liquid marbles were then transported to a 96-well plate containing $100 \mu \mathrm{L}$ of culture media. The media liquid assists the marbles to float and contribute the necessary nutrients during the harvesting step. Floating liquid marbles allow the cells to move freely and interact with other cells to produce uniform spheroids. The marble was incubated 
for 24 hours at $37^{\circ} \mathrm{C}$ and monitored with an optical-fluorescent microscope (Olympus IX70) to examine the cell survival as well as the formation of the spheroids.

\section{Injection of the spheroids}

Once multiple spheroids with average diameters ranging between 80 and $120 \mu \mathrm{m}$ were formed inside the liquid marble, they were harvested using centrifugal separation at a relatively low rotational speed of $500 \mathrm{rpm}$ for 5 minutes without disrupting the spheroidal structures. Debris including the PTFE powder and the matured media was removed. Fresh OEC media were then mixed with the spheroids as the base fluid to inject into the device. A number of marbles (between 10-15) each containing 15-25 spheroids were harvested for the injection. DI water was used to flush the device to remove any residual waste before spheroid seeding. Fluid injection through the microfluidic device was done by pipetting.

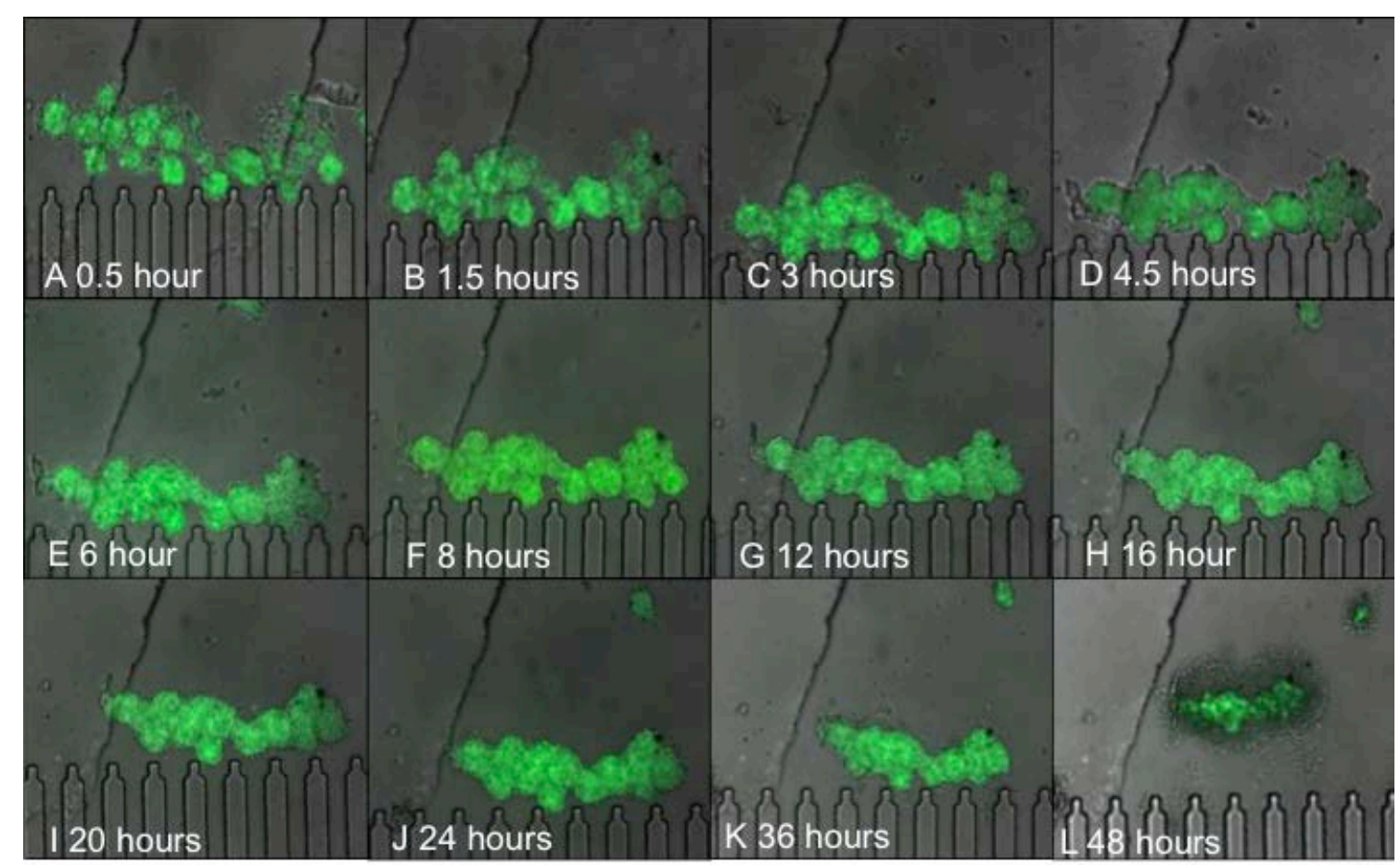

Fig. 7 OECs spheroids fused inside of the microfluidic chamber cultured up to 48 hours.

As the spheroids are very sensitive to pressure, the injection rate of $30 \mu \mathrm{L} / \mathrm{min}$ was attempted so that the majority of OEC spheroids could be trapped above the microchannel array. Initially, the culture media well was closed to create sufficient pressure inside the culture chamber for the trapping mode. A number of spheroids were trapped on the channel array as a result of hydrodynamic force. However, numerous spheroids (approximately half) still floated around in the chamber. The trapped spheroid with closed well setup did not promote the fusion phenomena due to the insufficient supply of nutrient and oxygen over the culture period (Fig S2).

In the fusion mode, we filled the well with fresh culture media that could supply sufficient nutrients to the spheroid. The LOC device was placed vertically inside of an incubator for 30 min. Gravitational force helped the media to fill the chamber and allowed the residual 
floating spheroids to settle down on the microchannel array. An average of 180 out of 200 spheroids corresponding to an efficiency of almost $90 \%$ were trapped on the channel array. Images were taken with fluorescent microscope and confirmed that most spheroids were trapped. The well was filled with fresh media for every 4 hours before the imaging process.

\section{Results and discussion}

Optical fluorescence microscopy was used to observe the fusion phenomena of OEC spheroids inside the micro-fluidic chamber. Figure 6 shows that most spheroids tended to settle in regions with the lowest velocity and pressure. Moreover, some of the already trapped spheroids near the inlet region were dragged and trapped further right, unblocking the microchannel on the left.

Fig. 6 depicts the fusion process of the OEC spheroids over a time period of 48 hours. Images were taken at an interval of 4 hours after removing the device from the incubation chamber and supplying fresh media to the well. Fig. 6A shows the trapped spheroids after 1 hour of incubation. Although the spheroids were still loose, they touched each other and were ready to fuse over time. Fig. 6B illustrates the same spheroids after 4 hour of incubation. Most spheroids now have interacted and moved slowly toward aggregations. Loose individual spheroids are difficult to be recognized now. Fig. 6C depicts the image taken after 8 hours of incubation. Clear fusion of the spheroids can be observed. Most of the spheroids were merged so that individual spheroids were no longer visible.

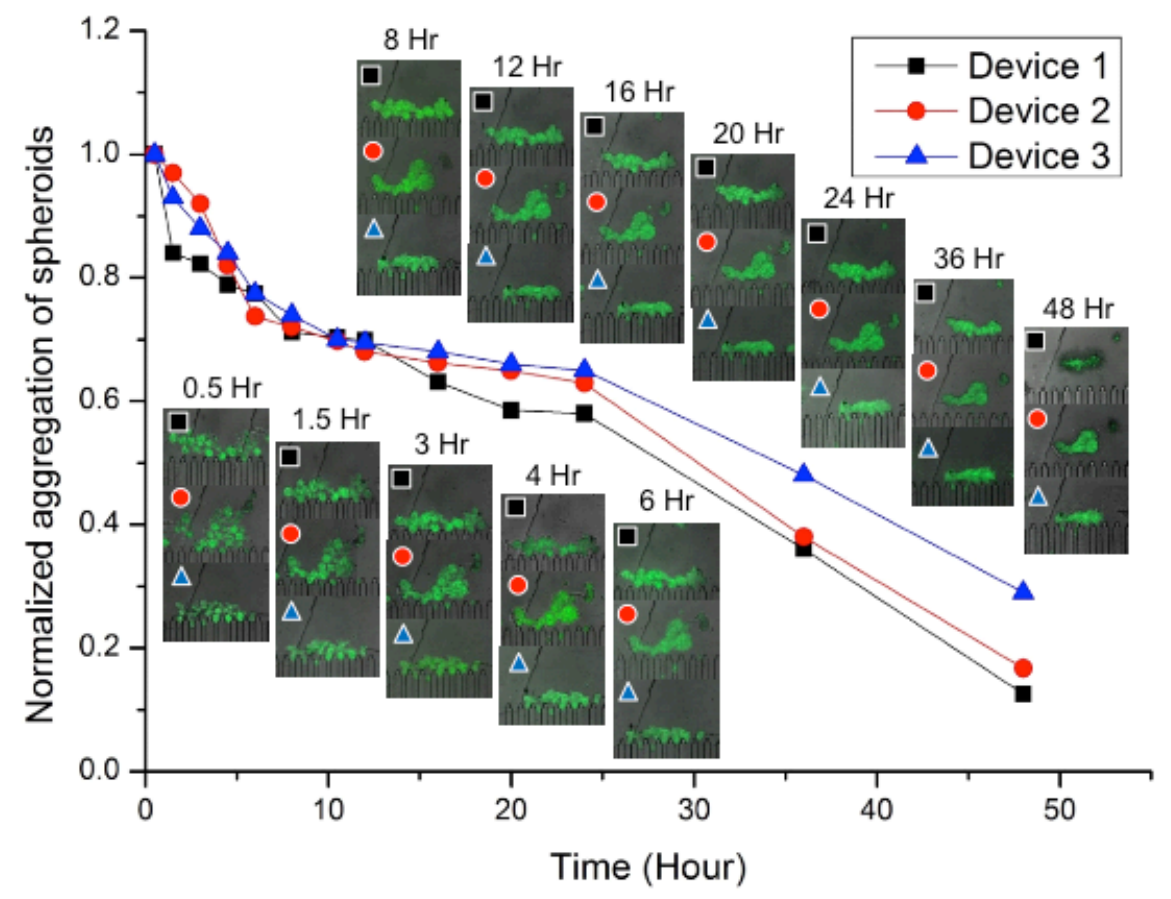

Fig. 8 Normalized aggregated spheroids over time within three LOC devices.

OEC spheroids showed extensive cell-to-cell interaction in all directions allowing for a healthy survival up to 12 hours. Fig. 6D indicates the stable physical structure of the formed tissue after 12 hours of culture. However, a slight narcosis of cells commenced was visible at 
the outer edges of the tissue in Fig. 6E. The length of the aggregation between multiple spheroids decreases much faster after 24 hours of incubation as shown in Figs. 6G to $6 \mathrm{H}$. After 24 hours culture, the merged spheroids behave like a tissue and tend to shrink. It is apparent that the spheroidal movements were strongly influenced by the presence of neighbouring spheroids. Closely placed OEC spheroids formed a thick confluent tissue layer. Our experiment validated that a small tissue engineered construct can be fabricated inside a microfluidic chamber from a number of fused spheroids. ${ }^{25}$

Fig. 7 shows the representative repeatability test of the OEC spheroids fusion process inside different LOC devices. The image was taken from the end region of the culture chamber where the OECs face minimum hydrodynamic forces. The image were taken at an interval of 1.5 to 2.0 hours up to 12 hours and cultured up to 48 hours (Fig S3-S5). Individual spheroids were visualized until 3 hours of incubation periods in Figs 7A to 7C. From 4.5 to 12 hours of incubation (Figs 7D to 7G). Individual spheroids were no longer identified, and tissue-like structures were observed. Cell narcosis were visible all the cases from 16 to 36 hour of incubation in the LOC devices (Fig. S3-S5). The aggregation rate is much faster after the 24 hour of cell culturing.

Fig. 8 shows the normalized aggregation rate of the cultured spheroids over time in different LOC devices. The normalized aggregation is defined as the ratio between the area of the cells to its initial value at the start of the experiment. The area of the large and identical spheroids (trapped at the end region with similar size) were analysed with ImageJ software (NIH, imagej.net). The aggregation size remains almost constant between 12 and 24 hours of incubation, indicating the fused tissue is stable and healthy and suitable for harvest. Afterwards the size decreases at a high rate due to necrosis, and a variation is observed at 48 hours of incubation. For the transplantation applications, the fused tissue should be harvested at around 12 hours of incubation, before cell necrosis occurs. Thus, the necrosis of the cell and the variation of the aggregation rate would not be an issue

The observed fusion phenomena can be explained by the Differential Adhesion Hypothesis (DAH). ${ }^{19,}{ }^{26}$ According to this hypothesis, cells possess a highly viscous incompressible liquid-like behaviour caused by the surface and interfacial tension generated by the adhesive and cohesive interactions between the cell. ${ }^{27}$ In the absence of an external force, a liquid droplet forms a spherical shape due to the attraction of its own molecules, allowing a maximum contact and minimum overall surface area. The spheroid has characteristics similar to highly viscous liquid droplets. Both of them consists of a large number of mobile, cohesive subunits: cells for spheroids versus molecules for droplets. These subunits spontaneously rearrange among themselves to maximize their mutual and relative bonding. Fusion is the self-assembly process where the liquid like cell matrix rearrange themselves towards a larger spherical form minimizing the free energy of the system. ${ }^{28}$ However, a major difference between a highly viscous droplet and a spheroid is that molecules in a droplet move due to the thermal agitations whereas cellular motion is governed by the metabolic energy. For spheroids, coalescence is due to the metabolic energy promoted by the protein obtained both from media and other cells. ${ }^{29}$ Throughout the fusion process, a morphogenetic shape changes occurs which is a coordinated movement of cells within individual spheroids. 


\section{Conclusion}

We have developed a microfluidic device that enables injection and capture of spheroids of OECs. Trapping spheroids at regularly spaced intervals enables them to fuse to form larger aggregations. Numerical modelling indicated that lower flow rates favour trapping of the spheroids. Visualisation of the flow field with fluorescent particles confirmed that the right side of the chamber has the lowest flow velocity and will likely trap the spheroids. The later injection of spheroids of OECs with diameters ranging from 80 and $120 \mu \mathrm{m}$ confirmed that the spheroids were trapped on the right side above the microchannel array. However, a number of spheroids continued to float above the trapping zone. The trapped spheroids commenced fusing within 4 hours of incubation. Large fused tissues were evident after 8 hours culturing. We were able to maintain multiple healthy OECs spheroids inside of a micro-chamber for up to 12 hours. Adding fresh media through the well promotes fusion and longer culture resulted in poor cell growth on the exterior of the fused spheroids. The fused spheroids can be harvester through the open well by reverse flowing the fluid from the outlet ports, keeping the inlet closed. The development of cell transplantation therapies needs new tools to improve the preparation of cells prior to transplantation into injured parts of human body. Our microfluidic device facilitating the fusion of OEC spheroids is a step towards this direction. As OECs spheroids exhibits a higher degree of cell-cell and cell-matrix interaction than preparations of dispersed cell suspensions, spheroidal transplantation would be more effective for the neural repair therapies as it would promote the integration of the cells within the host tissue. This microfluidic device could be used to determine the cellular and molecular mechanisms of cell spheroid fusion to identify factors that enhance the rate of fusion. Furthermore, the microfluidic device presented here may be utilized for examining the effect of drug responses, improving therapeutic approaches.

\section{Acknowledgement}

This work was supported by a grant from the Perry Cross Spinal Research Foundation and a grant from the Clem Jones Foundation to JSJ. The fabrication of the devices was performed in part at the Queensland Node of the Australian National Fabrication Facility, a company established under the National Collaborative Research Infrastructure Strategy to provide nano and micro- fabrication facilities for Australia's researchers.

\section{References}

1. R.-H. Chou, C.-Y. Lu, J.-R. Fan, Y.-L. Yu and W.-C. Shyu, Cell transplantation, 2014, 23, 567-571.

2. H. Yang, B.-R. He and D.-J. Hao, Molecular neurobiology, 2015, 51, 168-179.

3. Y. Zhu, L. Cao, Z. Su, L. Mu, Y. Yuan, L. Gao, Y. Qiu and C. He, Glia, 2010, 58, 716-729.

4. R. Doucette, International journal of developmental neuroscience, 1996, 14, 945-959.

5. $\quad$ E. Woodhall, A. K. West and M. I. Chuah, Molecular brain research, 2001, 88, 203213.

6. P. Tabakow, W. Jarmundowicz, B. Czapiga, W. Fortuna, R. Miedzybrodzki, M. Czyz, J. Huber, D. Szarek, S. Okurowski and P. Szewczyk, Cell transplantation, 2013, 22, 1591-1612. 
7. R. K. Vadivelu, C. H. Ooi, R.-Q. Yao, J. T. Velasquez, E. Pastrana, J. Diaz-Nido, F. Lim, J. A. Ekberg, N.-T. Nguyen and J. A. St John, Scientific reports, 2015, 5.

8. G. F. Chi, M.-r. Kim, D.-W. Kim, M. H. Jiang and Y. Son, Experimental neurology, 2010, 222, 304-317.

9. T.-C. Tseng and S.-h. Hsu, Biomaterials, 2014, 35, 2630-2641.

10. G. S. Jeong, J. Y. Chang, J. S. Park, S.-A. Lee, D. Park, J. Woo, H. An, C. J. Lee and S.-H. Lee, Molecular brain, 2015, 8, 1.

11. B. A. Justice, N. A. Badr and R. A. Felder, Drug discovery today, 2009, 14, 102-107.

12. S. Breslin and L. O’Driscoll, Drug discovery today, 2013, 18, 240-249.

13. M. Bokhari, R. J. Carnachan, N. R. Cameron and S. A. Przyborski, Biochemical and biophysical research communications, 2007, 354, 1095-1100.

14. F. Sarvi, T. Arbatan, P. P. Y. Chan and W. Shen, Rsc Advances, 2013, 3, 1450114508.

15. C. H. Ooi and N.-T. Nguyen, Microfluidics and Nanofluidics, 2015, 19, 483-495.

16. K. C. Bhargava, B. Thompson and N. Malmstadt, Proceedings of the National Academy of Sciences, 2014, 111, 15013-15018.

17. G. M. Whitesides, Nature, 2006, 442, 368-373.

18. F. Zhu, J. Skommer, N. P. Macdonald, T. Friedrich, J. Kaslin and D. Wlodkowic, Biomicrofluidics, 2015, 9, 046502.

19. M. S. Steinberg, Current opinion in genetics \& development, 2007, 17, 281-286.

20. V. Beachley, V. Kasyanov, A. Nagy-Mehesz, R. Norris, I. Ozolanta, M. Kalejs, P. Stradins, L. Baptista, K. da Silva and J. Grainjero, Journal of tissue engineering, 2014, 5, 2041731414556561.

21. A. Munaz, R. K. Vadivelu, J. S. John, M. Barton, H. Kamble and N.-T. Nguyen, Journal of Science: Advanced Materials and Devices, 2016, 1, 1-17.

22. J. Fukuda and K. Nakazawa, Biomicrofluidics, 2011, 5, 022205.

23. H. Ota, R. Yamamoto, K. Deguchi, Y. Tanaka, Y. Kazoe, Y. Sato and N. Miki, Sensors and Actuators B: Chemical, 2010, 147, 359-365.

24. T. Anada, T. Masuda, Y. Honda, J. Fukuda, F. Arai, T. Fukuda and O. Suzuki, Sensors and Actuators B: Chemical, 2010, 147, 376-379.

25. Z. Hajdu, V. Mironov, A. N. Mehesz, R. A. Norris, R. R. Markwald and R. P. Visconti, Journal of tissue engineering and regenerative medicine, 2010, 4, 659-664.

26. R. A. Foty and M. S. Steinberg, Developmental biology, 2005, 278, 255-263.

27. G. Forgacs, R. A. Foty, Y. Shafrir and M. S. Steinberg, Biophysical journal, 1998, 74, 2227-2234.

28. P. A. Fleming, W. S. Argraves, C. Gentile, A. Neagu, G. Forgacs and C. J. Drake, Developmental Dynamics, 2010, 239, 398-406.

29. E. Flenner, F. Marga, A. Neagu, I. Kosztin and G. Forgacs, Current topics in developmental biology, 2008, 81, 461-483. 